\title{
Isotopic fractionation during grain coarsening of wet snow
}

\author{
MaSAYOSHI NAKAWO*, \\ Nagaoka Institute of Snow and Ice Studies, NIED, Suyoshi, Nagaoka, Niigata 940, Japan \\ SHIGERU CHIBA, \\ Department of Applied Physics, Faculty of Engineering, Hokkaido University, Sapporo 060, Japan \\ Hiroshi SATAKe AND SHigeru Kinouchi \\ Department of Earth Sciences, Faculty of Science, Toyama University, Toyama 930, Japan
}

\begin{abstract}
Isotopic composition of solid and liquid portions of wet snow was investigated experimentally. The compositions changed with time, $\delta$ values of ice becoming heavier than those for water. A simple model was proposed to explain their temporal variation. It predicted, however, a more rapid change of $\delta$ values than the trend obtained in the experiments. This suggests the presence of a "diffusion layer" adjacent to growing snow particles, where isotope concentration has dropped at the ice-water interface because of the fractionation during grain coarsening. The slope in $\delta \mathrm{D}-\delta^{18} \mathrm{O}$ diagram estimated by the model is compatible with the experimental data. It is considered, therefore, that the freezing fraction, the part of the liquid which refreezes to relatively large particles during grain coarsening, could be estimated by measuring the isotope concentration.
\end{abstract}

\section{INTRODUCTION}

Isotope concentration in polar snow and ice is considered to be a good indicator of temperature, and many workers have reconstructed past temperature records at various places in Greenland and Antarctica by looking at the concentration change along ice cores (e.g. Dansgaard and others, 1973; Lorius and others, 1985). This method has been extended to ice cores taken at rather warm sites where snow particles in surface layers are subjected to wet-snow metamorphism for a significant period of time.

In wet snow metamorphism, the rate of grain coarsening is much faster than in dry metamorphism (e.g. Wakahama, 1968). This is mainly due to melting of small particles and freezing of surrounding liquid water on to large particles, and the major portion of their volume has been slowly frozen from the liquid phase at an advanced stage (Raymond and Tusima, 1979). Isotope concentration of snow particles as well as surrounding water evolves considerably during wet-snow metamorphism, since isotopic fractionation takes place with the phase changes. Búason (1972) examined the isotopic fractionation in a melting snow column by assuming an

* Present address: Institute for Hydrospheric-Atmospheric Sciences, Nagoya University, Nagoya 464-01, Japan. exponential decay of fractionation with time. It is important to know how isotopic fractionation takes place during grain coarsening in wet snow.

This paper aims at investigating quantitatively the isotopic fractionation in wet-snow metamorphism, and at providing basic knowledge for interpretation of isotope data collected from ice cores from relatively warm areas.

\section{NOTATION}

$\alpha \quad$ fractionation factor

$\alpha \mathrm{D}$ fractionation factor for deuterium, 1.0206

(Suzuoki and Kimura, 1973)

$\alpha^{18} \mathrm{O}$ fractionation factor for ${ }^{18} \mathrm{O}, 1.0028$

(Suzuoki and Kimura, 1973)

$\delta \quad$ isotopic composition denoted with a reference of Standard Mean Ocean Water (SMOW):

$\delta=\left(a_{\text {sample }}-a_{\text {SMOW }}\right) / a_{\text {SMOW }}$, where $a$ is the isotopic ratio, $D / H$ or ${ }^{18} \mathrm{O} /{ }^{16} \mathrm{O}$

$\delta \mathrm{D} \delta$ value for deuterium

$\delta^{18} \mathrm{O} \quad \delta$ value for ${ }^{18} \mathrm{O}$

$\delta_{\mathrm{i}} \quad \delta$ value for freezing ice

$\delta_{\mathrm{iz}} \quad \delta$ value for total ice portion (snow particles)

$\delta_{\mathrm{w}} \quad \delta$ value for water

$\delta_{\text {io }} \quad \delta$ value for original snow particles

$\delta_{\text {wo }} \delta$ value for original water

$f \quad$ fraction of freezing (or melting) 


\section{EXPERIMENT}

Sieved snow kept in a $0^{\circ} \mathrm{C}$ chamber (a container surrounded by snow-water mixture) was mixed with an equal amount of water also stored in the chamber. The wet snow of $50 \%$ water content thus prepared was bottled in $100 \mathrm{cc}$ plastic containers; these were submerged in a large container of snow jam (water-rich wet snow), which was placed in the $0^{\circ} \mathrm{C}$ chamber. The whole system was kept in a cold room at a temperature of $0^{\circ} \pm 2^{\circ} \mathrm{C}$.

The samples were analyzed in a series of experiments. First a subsample from a bottle was photographed under a microscope for analyzing particle size distribution. The rest of the sample was then centrifuged to separate liquid and solid portions.

Pre-experiments were conducted to determine how long the centrifuge should be operated to achieve complete separation. Wet-snow samples prepared as above were subjected to separation for certain time periods, and the water content of the resultant solid portion of each sample was measured with a calorimetric method having an accuracy of $\pm 1 \%$ (Akitaya, 1978). The water content decreased as the time period increased, as shown in Figure 1. The scatter in water content before separation began indicates that $60-80 \%$ of the liquid water between the snow particles drained off naturally when the sample was placed in a container. By rotating the separator, most of the rest of the water was extracted from the sample. The water content of the sample stabilized at $\sim 3 \%$ after several minutes' rotation.

In each experimental run the liquid and the solid portions were subjected to the isotope analyses after ten minutes of separation. Their $\delta^{18} \mathrm{O}$ values were measured using the $\mathrm{CO}_{2}$ equilibration method (Yoshida and Mizutani, 1986). Some portion of each sample was converted to hydrogen gas by hot uranium, and its deuterium concentration $\delta \mathrm{D}$ was measured with a mass spectrometer, Micro Mass $602 \mathrm{E}$. The $\delta^{18} \mathrm{O}$ and $\delta \mathrm{D}$ values of the solid ice portion $\left(\delta^{18} \mathrm{O}_{\mathrm{iz}}\right.$ and $\delta \mathrm{D}_{\mathrm{iz}}$ respectively) were calibrated with the values for the liquid portion $\left(\delta^{18} \mathrm{O}_{\mathrm{w}}\right.$ and $\delta \mathrm{D}_{\mathrm{w}}$ ) assuming the $3 \%$ liquid-water content established in the pre-experiments.

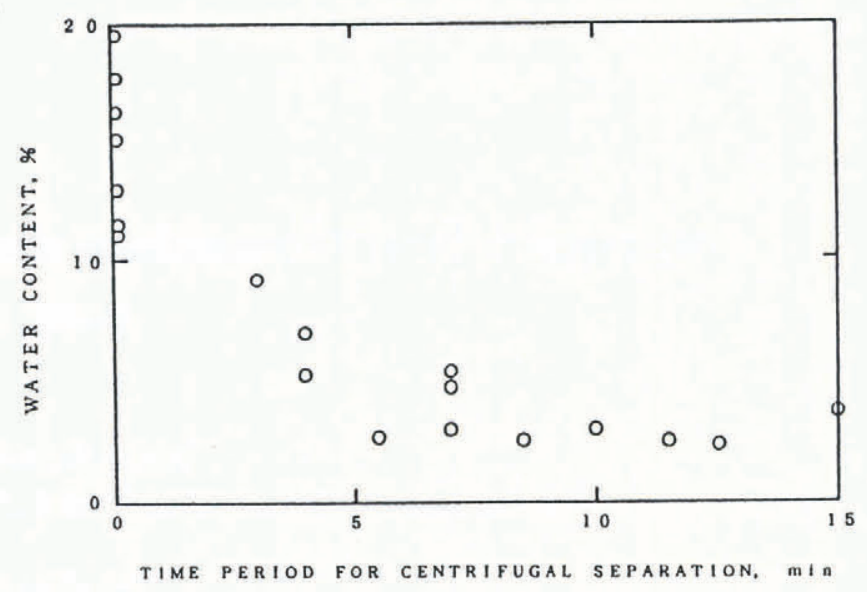

Fig. 1. Water content change of wet-snow sample by centrifugal separation.

Four experimental runs were carried out, as shown in Table 1 . In runs B and C, isotopically heavier snow from Sapporo, Japan, was mixed with lighter water prepared by melting Antarctic snow. The electrical conductivity of the snow collected at Sapporo is $10-100 \mu \mathrm{S} \mathrm{cm}^{-1}$ (Suzuki, 1987), and the Antarctic snow has conductivity of 1$10 \mu \mathrm{S} \mathrm{cm}^{-1}$. In run $\mathrm{A}$, Antarctic snow was mixed with distilled and de-ionized tap water with electrical conductivity of $<\left(6 \times 10^{-2}\right) \mu \mathrm{S} \mathrm{cm}^{-1}$. In run $\mathrm{D}$, an $\mathrm{NaCl}$ solution of $\left(8 \times 10^{-4}\right) \mathrm{mol} \mathrm{kg}^{-1}$ was mixed with Antarctic snow. In runs $A$ and $D$, therefore, the snow was isotopically lighter than the water prepared from tap water at Nagaoka, Japan.

Ion concentration of the initial snow or water samples was highest in the $\mathrm{NaCl}$ solution and second highest in Sapporo snow. Raymond and Tusima (1979) found, however, that the rate of grain coarsening was not affected by the presence of impurities when their concentration was $<0.01 \mathrm{~mol} \mathrm{~kg}^{-1}$. The present study also showed no discernible difference between the different runs in the temporal change of the particle size distribution, which will be discussed later.

Table 1. Experiments ( $\delta$ values are shown in parts per thousand)
Run
$\delta \mathrm{D}_{\text {io }} \quad \delta^{18} \mathrm{O}_{\text {io }} \quad \delta \mathrm{D}_{\text {wo }} \quad \delta^{18} \mathrm{O}_{\text {wo }}$
Remarks

$\begin{array}{lrrrr}\text { A } & -144.0 & -18.9 & -68.2 & -11.0 \\ \text { B } & -53.6 & -10.8 & -144.0 & -18.9 \\ & & & & \\ \text { C } & -75.4 & -10.9 & -150.0 & -20.0 \\ \text { D } & -153.2 & -20.2 & -58.1 & -10.0\end{array}$

Antarctic snow collected near Syowa Station combined with distilled and de-ionized tap water from Nagaoka, Japan

Snow sample collected at Sapporo, Japan, combined with melted water from Antarctic snow (collected near Syowa Station)

Sapporo snow combined with water from melted Antarctic snow

Antarctic snow combined with $\mathrm{NaCl}$ solution of $8.1 \times 10^{-4} \mathrm{~mol} \mathrm{~kg}^{-1}$ prepared with Nagaoka distilled and de-ionized tap water 


\section{SLOPE OF $\delta \mathrm{D}-\delta^{18}$ O DIAGRAM}

Grain coarsening of wet snow has been explained in terms of heat-flow controlled melting and refreezing determined by the curvature of particle surfaces (Raymond and Tusima, 1979). Under an isothermal conditon at $0^{\circ} \mathrm{C}$, the amount of melting of relatively small particles is identical with the amount of freezing of water on to large particles. The $\delta$ values for the total ice portion is hence given by

$$
\delta_{\mathrm{iz}}=\delta_{\mathrm{io}}(1-f)+\int_{0}^{f} \delta_{\mathrm{i}} \mathrm{d} f .
$$

Because of the fractionation of heavy isotopes during freezing,

$$
\alpha\left(1+\delta_{\mathrm{w}}\right)=1+\delta_{\mathrm{i}} .
$$

The mass conservation law gives

$$
\frac{\mathrm{d}}{\mathrm{d} f}\left(\delta_{\mathrm{iz}}+\delta_{\mathrm{w}}\right)=0 .
$$

From Equations (1), (2) and (3)

$$
\begin{gathered}
\delta_{\mathrm{i}}=\delta_{\mathrm{io}}-\exp (-\alpha f)\left[\left(\delta_{\mathrm{io}}+1\right)-\alpha\left(\delta_{\mathrm{wo}}+1\right)\right], \\
\delta_{\mathrm{iz}}=\delta_{\mathrm{io}}+\frac{1}{\alpha}\left[\left(\delta_{\mathrm{io}}+1\right)-\alpha\left(\delta_{\mathrm{wo}}+1\right)\right][\exp (-\alpha f)-1],
\end{gathered}
$$

and

$$
\delta_{\mathrm{w}}=\delta_{\mathrm{wo}}+\frac{1}{\alpha}\left[\left(\delta_{\mathrm{io}}+1\right)-\alpha\left(\delta_{\mathrm{wo}}+1\right)\right][1-\exp (-\alpha f)] .
$$

Equations (5) and (6) hold for either deuterium or ${ }^{18} \mathrm{O}$, and

$$
\frac{\delta \mathrm{D}_{\mathrm{iz}}-\delta \mathrm{D}_{\mathrm{io}}}{\delta^{18} \mathrm{O}_{\mathrm{iz}}-\delta^{18} \mathrm{O}_{\mathrm{io}}}=\frac{\delta \mathrm{D}_{\mathrm{w}}-\delta \mathrm{D}_{\mathrm{wo}}}{\delta^{18} \mathrm{O}_{\mathrm{w}}-\delta^{18} \mathrm{O}_{\mathrm{wo}}}=K \cdot g(f),
$$

where

$$
K=\frac{\left[\left(\delta \mathrm{D}_{\mathrm{io}}+1\right)-\alpha \mathrm{D}\left(\delta \mathrm{D}_{\text {wo }}+1\right)\right]}{\left[\left(\delta^{18} \mathrm{O}_{\mathrm{io}}+1\right)-\alpha^{18} \mathrm{O}\left(\delta^{18} \mathrm{O}_{\text {wo }}+1\right)\right]}
$$

and

$$
g(f)=\left(\frac{\alpha^{18} \mathrm{O}}{\alpha \mathrm{D}}\right) \frac{1-\exp (-\alpha \mathrm{D} f)}{1-\exp \left(-\alpha^{18} \mathrm{O} f\right)}
$$

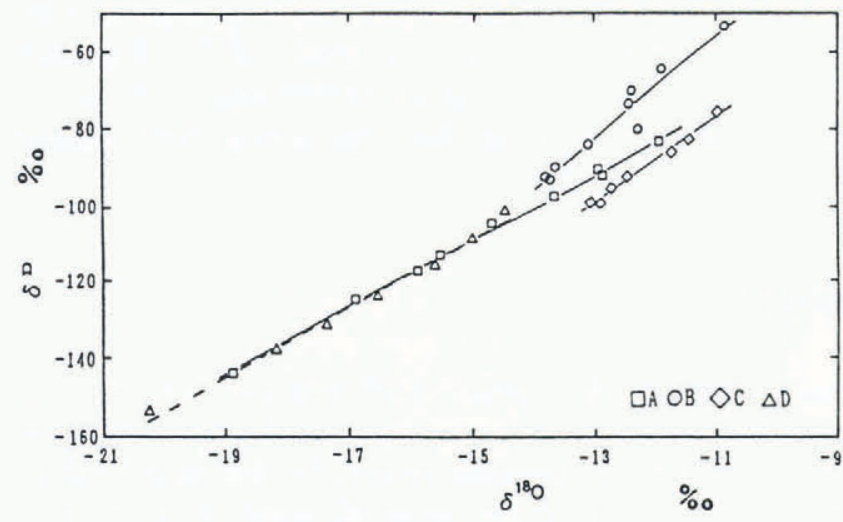

Fig. 2. $\delta D-\delta^{18} O$ diagram for ice portion (snow particles). Straight lines are linear regressions.

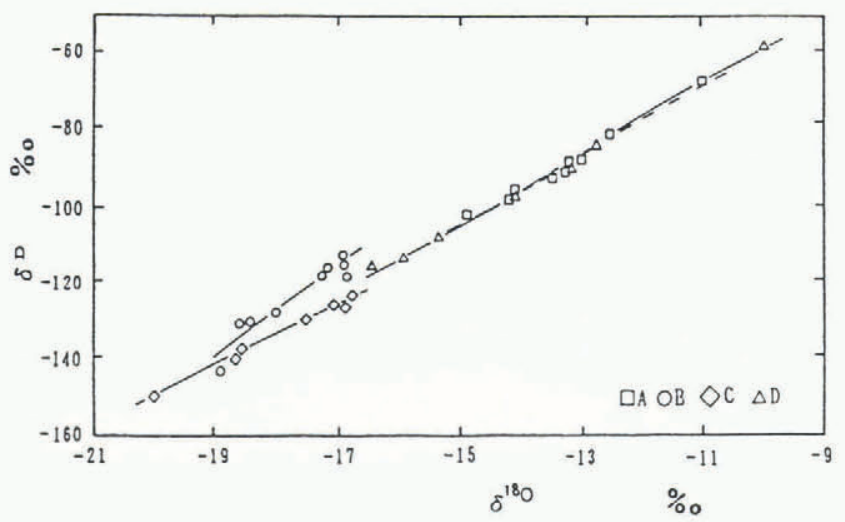

Fig. 3. $\delta D-\delta^{18} O$ diagram for liquid water. Straight lines are linear regressions.

Equation (7) gives a slope in $\delta \mathrm{D}-\delta^{18} \mathrm{O}$ diagram as shown in Figures 2 and 3, where experimental data are plotted. Straight lines in the figures are linear regressions for each run of the experiment, and their slopes are given

\begin{tabular}{|c|c|c|c|}
\hline$K$ & 8.91 & 13.70 & 9.07 \\
\hline$K \cdot \overline{g(f)}$ & 8.88 & 13.64 & 9.03 \\
\hline
\end{tabular}
in Table 2.

Table 2. Slopes in $\delta D-\delta^{18} O$ diagram, $(\overline{g(f)}$ is the average for $f=0$ to 1 )

$\begin{array}{llllll}\text { Run } & A & B & C & D\end{array}$

Data regression

$\begin{array}{llrrr}\delta_{\mathrm{iz}} & 8.68 & 13.12 & 10.59 & 8.84 \\ \delta_{\mathrm{w}} & 8.82 & 12.18 & 7.90 & 9.12 \\ \text { average } & 8.75 & 12.65 & 9.25 & 8.98\end{array}$

Calculation by

Equation (6)

The function $g(f)$ is a monotonously decreasing function from 1 for $f=0$ to 0.99 for $f=1$, which, therefore, can be practically regarded as 1 . The slope of $\delta$ change in $\delta \mathrm{D}-\delta^{18} \mathrm{O}$ diagram is hence essentially dependent on $K$, a function of initial $\delta$ values. The $K$ values for different runs are given in Table 2 in association with the values for $K \cdot \overline{g(f)}$, where $\overline{g(f)}$ is the average of $g(f)$ for $f=0$ to 1 . No significant difference can be seen between the values of $K$ and $K \cdot \overline{g(f)}$, as mentioned above.

The calculation of the slope by Equation (7) compares favourably with those derived by regression analysis. This indicates that we would be able to estimate the change in isotopic composition through wet snow metamorphism, when we know $\delta$ values for both the initial snow and water components. The slope given by Equation (7) is different from those derived by Souchez and Jouzel (1984) for successive freezing of water in a closed system. 


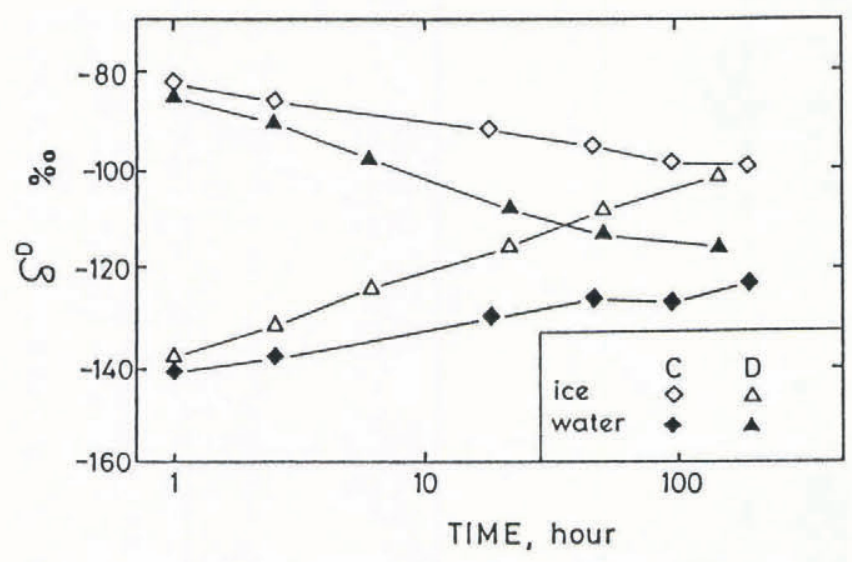

Fig. 4. Examples of temporal change of $\delta$ values.

\section{FRACTIONATION WITH TIME}

Examples of temporal changes in $\delta_{\mathrm{iz}}$ and $\delta_{\mathrm{w}}$ are shown in Figure 4. In both runs C and D the $\delta$ values of ice and water changed, approaching each other with time. In run $\mathrm{D}$, where ice was originally lighter than water, the $\delta$ values crossed and ice became heavier than water at an advanced stage. On the other hand, in run $\mathrm{C}$ where ice was heavier than water when the experiment started, the rate of $\delta$ change was more gentle and the $\delta$ values appeared stable after a certain period. This feature is considered reasonable, since ice should be heavier than water at the equilibrium state.

Raymond and Tusima (1979) examined in detail the evolution of the particle size distribution of watersaturated snow. They proposed the following equation to represent the normalized cumulative frequency curve for the size distribution during the grain coarsening of wet snow:

$$
\psi^{*}\left(\frac{v}{v_{\mathrm{m}}}\right)=\left(1-\frac{a v}{\mathrm{~b} v_{\mathrm{m}}}\right)^{\frac{1}{\mathrm{a}}},
$$

where $v$ is the volume of a particle, $v_{\mathrm{m}}$ is the median volume, and $\mathrm{a}$ and $\mathrm{b}$ are constants to be 0.23 and 1.55 , respectively. It predicts zero probability for particles larger than a maximum volume of $v_{\mathrm{m}} \mathrm{b} / \mathrm{a}$. Equation (8) fits nicely to our data, as shown in Figure 5, except for

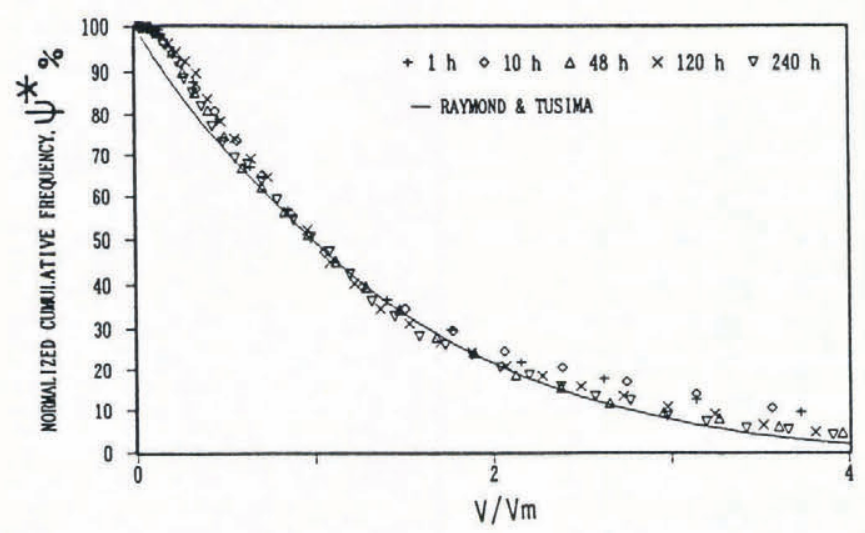

Fig. 5. Example of particle size distribution plotted as function of $v / v_{m}$. small sizes, say $v / v_{\mathrm{m}}<0.5$ and for the relatively early stage. The frequency curve $\phi^{*}$ is then given by differentiating Equation (8), hence

$$
\phi^{*}\left(\frac{v}{v_{\mathrm{m}}}\right)=\frac{1}{\mathrm{~b} v_{\mathrm{m}}}\left(1-\frac{\mathrm{a} v}{\mathrm{~b} v_{\mathrm{m}}}\right)^{\frac{1-\mathrm{a}}{\mathrm{a}}} .
$$

Raymond and Tusima (1979) derived also the freezing rate $S(v, t)$ for a particle with the volume $v$ :

$$
S(v, t)=\frac{\mathrm{d} v_{\mathrm{m}}}{\mathrm{d} t}\left[(1+\mathrm{a}) \frac{v}{v_{\mathrm{m}}}-b\right],
$$

where $t$ is time. Taking $V$ to be the total volume and $N$ to be the the number of snow particles in the system under consideration, the rate of increase in freezing fraction for the total snow particles is given by

$$
\frac{\mathrm{d} f}{\mathrm{~d} t}=\frac{1}{V} \int_{b v_{\mathrm{m}} /(1+\mathrm{a})}^{b v_{\mathrm{m}} / \mathrm{a}} N(t) \cdot \phi^{*}\left(\frac{v}{v_{\mathrm{m}}}\right) \cdot S(v, t) \mathrm{d} v,
$$

since the grain coarsening takes place only for particles larger than the average grain volume, which is expressed by $b v_{\mathrm{m}} /(1+\mathrm{a})$ (Raymond and Tusima, 1979). Substitution of Equations (9) and (10) into Equation (11), and performing the integration gives the following equation, taking into account that $N(t)$ is given by $((1+\mathrm{a}) V) /\left(\mathrm{b} V_{\mathrm{m}}\right):$

$$
f=\beta \ln \left(1+\frac{\gamma}{v_{0}} t\right),
$$

where $\beta=(1 /(1+\mathrm{a}))^{1 / \mathrm{a}}, \gamma=\mathrm{d} v_{\mathrm{m}} / \mathrm{d} t$, and $v_{0}$ is the initial median volume.

Substitution of Equation (12) into Equations (5) and (6) gives $\delta_{\mathrm{iz}}$ or $\delta_{\mathrm{w}}$ as a function of time. When $\delta_{\mathrm{iz}}$ and $\delta_{\mathrm{w}}$ are normalized,

$$
\begin{aligned}
\frac{\left(\delta_{\mathrm{iz}}-\delta_{\mathrm{io}}\right) \alpha}{\left[\left(\delta_{\mathrm{io}}+1\right)-\alpha\left(\delta_{\mathrm{wo}}+1\right)\right]} & =\frac{-\left(\delta_{\mathrm{w}}-\delta_{\mathrm{wo}}\right) \alpha}{\left[\left(\delta_{\mathrm{io}}+1\right)-\alpha\left(\delta_{\mathrm{wo}}+1\right)\right]} \\
& =\left(1+\frac{\gamma}{v_{0}} t\right)^{-\alpha \beta}-1 .
\end{aligned}
$$

Equation (13) is plotted in Figure 6 for deuterium and in Figure 7 for ${ }^{18} \mathrm{O}$. Solid lines in the figures were calculated with the average value of $\gamma / v_{0}$ for all the experiments. Dashed lines are for the extreme values of $\gamma / v_{0}$ in the experiments. These curves are essentially the same in both figures, since the difference between $\alpha \mathrm{D}$ and $\alpha^{18} \mathrm{O}$ is very small.

Experimental data on $\delta_{\mathrm{iz}}$ and $\delta_{\mathrm{w}}$ are also plotted in Figures 6 and 7. It is found that the $\delta$ change is more gentle in the experiment than predicted by Equation (13). This indicates that at an earlier stage, the rate is faster than the prediction, since the normalized $\delta$ should be zero when $t$ is zero.

\section{DISGUSSION}

Snow particles at a certain grain-size grow in an early stage but they melt out in an advanced stage, when the average grain-size becomes larger. In the model calculation these particles are assumed, when they melt, to have the same isotopic concentration as the original snow, although they consist of the original snow portion plus the 


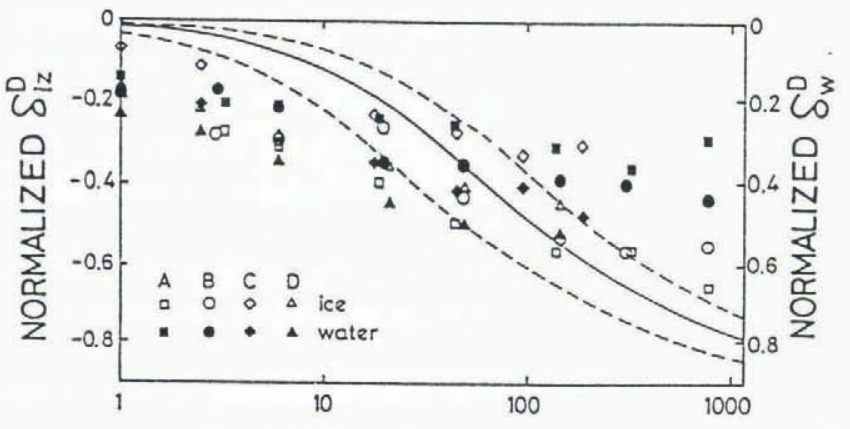

TIME, hour

Fig. 6. Evolution of normalized $\delta$ values for deuterium. Solid and dashed curves are given by Equation (13) for different values of $\gamma / v_{0}$.

newly frozen portion with different $\delta$ values. This, however, cannot be a major cause of the discrepancy between the data and the theory found in Figures 6 and 7, since the different combinations with initially heavier ice than water and with lighter ice than water gave similar results, as can be seen in the figures.

In deriving Equation (13), the following assumptions were made implicitly.

(1) Diffusion of the heavy isotopes can be neglected in the ice matrix.

(2) Liquid portion of wet snow is isotopically homogeneous: heavy isotopes diffuse very rapidly in water.

(3) Fractionation during grain coarsening is an equilibrium process and the fractionation factor has a constant value throughout the process.

The diffusion coefficient of the heavy isotopes is of the order of $10^{-14} \mathrm{~m}^{2} \mathrm{~s}^{-1}$ for ice at $0^{\circ} \mathrm{C}$ (Hobbs, 1974) and of $10^{-9} \mathrm{~m}^{2} \mathrm{~s}^{-1}$ for water near $0^{\circ} \mathrm{C}$ (Eisenberg and Kauzmann, 1969). The order of diffusion distance, for example, becomes $10^{-7} \mathrm{~m}$ in ice and $10^{-5} \mathrm{~m}$ in water, say in $1 \mathrm{~s}$. The speed of displacement of the solid-liquid interface through melting or freezing of snow particles was considered, based on the experiments, as having an order of $10^{-4} \mathrm{~m} \mathrm{~s}^{-1}$, which is much faster than the diffusion speed in the ice matrix. The first assumption, therefore, would be a reasonable one.

In liquid water, however, the diffusion speed is almost equivalent with or even smaller than the speed of interface movement owing to the grain coarsening. Isotope concentration of the water adjacent to growing grains, therefore, would become smaller because of the fractionation at the interface. After the initial stage of grain growth, this would lead to a slower rate in the temporal change of $\delta$ values for ice and water. The discrepancy between the data and the theory, therefore, could be due to the "diffusion layer" surrounding growing grains.

The $\delta$ values seem to change more rapidly than predicted by Equation (13) at an early stage of the process, as was mentioned in the previous section. A

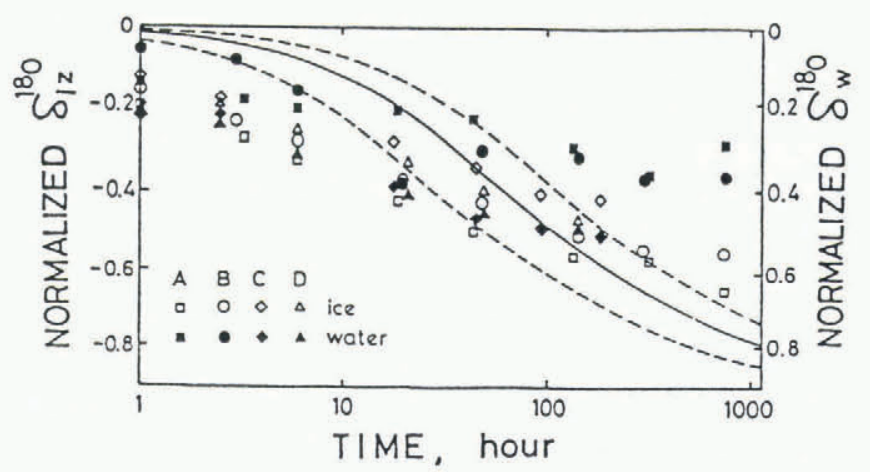

Fig. 7. Evolution of normalized $\delta$ values for ${ }^{18} O$. Solid and dashed curves are given by Equation (13) for different values of $\gamma / v_{0}$.

possible explanation for the difference is that the fractionation factor may not be a constant and could be larger, at an early stage of the process, than the value used in the calculation. It might depend on curvature. The equilibrium temperature at an ice-water interface is lower than $0^{\circ} \mathrm{C}$ when the snow particles are small, because of the curvature effect. The depression of the equilibrium temperature would decrease as the grain-size increases with time. At an early stage the fractionation factor can possibly be larger than that at a later stage, since it generally becomes large with decreasing temperature. The amount of the temperature depression caused by the curvature effect, however, is rather small, and may not be able to explain the discrepancy between the theory and the data at an early stage of the grain coarsening. Detailed study would have to be carried out to answer this question.

The model calculation of the temporal change of $\delta$ values for both the ice portion and the liquid portion was not very successful. The estimate of the slopes for $\delta \mathrm{D}-\delta^{18} \mathrm{O}$ diagram, however, was compatible with the experimental data, as mentioned above. This is attributed to the fact that the ratio of fractionation of deuterium to that of ${ }^{18} \mathrm{O}$ would not be affected by the rate of fractionation which could be controlled by the formation of the "diffusion layer" surrounding growing snow particles. Since the slope of the $\delta \mathrm{D}-\delta^{18} \mathrm{O}$ diagram can be estimated from the initial $\delta$ values of snow and water before mixing, the frozen fraction, the part of the liquid which refreezes to relatively large particles during grain coarsening, could possibly be estimated by measuring the isotopic composition, as was done by Jouzel and Souchez (1982) for regelation ice at a glacier bed, since the slope in wet snow metamorphism is different from 8 , the value in evaporation-condensation process (Craig and others, 1963).

\section{REFERENCES}

Akitaya, E. 1978. Measurements of free water content of wet snow by calorimetric method. Low Temp. Sci., Ser. A 36, 103-111. [In Japanese with English summary.]

Búason, T. 1972. Equation of isotope fractionation between ice and water in a melting snow column 
with continuous rain and percolation. 7. Glaciol., 11(63), 387-405.

Craig, H., L. I. Gordon and Y. Horibe. 1963. Isotopic exchange effects in the evaporation water. 1. Lowtemperature experimental results. F. Geophys. Res., 68(17), 5079-5087.

Dansgaard, W., S.J. Johnsen, H.B. Clausen and N. Gundestrup. 1973. Stable isotope glaciology. Medd. Grenl., 197(2).

Eisenberg, D. and W. Kauzmann. 1969. The structure and properties of water. Oxford, Clarendon Press.

Hobbs, P. V. 1974. Ice physics. Oxford, Clarendon Press.

Jouzel, J. and R. A. Souchez. 1982. Melting-refreezing at the glacier sole and the isotopic composition of the ice. 7. Glaciol., 28(98), 35-42.

Lorius, C. and 6 others. 1985. A 150,000-year climatic record from Antarctic ice. Nature, 316(6029), 591-596.

Raymond, C.F. and K. Tusima. 1979. Grain coarsening of water-saturated snow. 7. Glaciol., 22(86), 83-105.
Souchez, R.A. and J. Jouzel. 1984. On the isotopic composition in $\delta \mathrm{D}$ and $\delta^{18} \mathrm{O}$ of water and ice during freezing. F. Glaciol., 30(106), 369-372.

Suzuki, K. 1987. Spatial distribution of chloride and sulfate in the snow cover in Sapporo, Japan. Atmos. Environ., 21, 1773-1778.

Suzuoki, T. and T. Kimura. 1973. D/H and ${ }^{18} \mathrm{O} /{ }^{16} \mathrm{O}$ fractionation in ice-water system. Mass Spectroscopy, 21, 229-233.

Wakahama, G. 1968. The metamorphism of wet snow. International Association of Scientific Hydrology Publication 79 (General Assembly of Bern 1967 - Snow and Ice), 370379.

Yoshida, N. and Y. Mizutani. 1986. Preparation of carbondioxide for oxygen-18 determination of water by use of a plastic syringe. Anal. Chem., 58, 1273-1275.

The accuracy of references in the text and in this list is the responsibility of the authors, to whom queries should be addressed. 